\title{
Caracterização mineralógica de Latossolos em diferentes feições do relevo na região de Jaboticabal, $\mathrm{SP}^{1}$
}

\author{
Characterization mineralogical of Oxisol in different relief form in the region of \\ Jaboticabal, SP
}

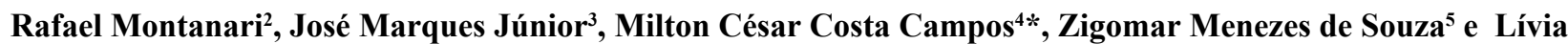 \\ Arantes Camargo 6
}

\begin{abstract}
Resumo - As diferentes feições da superfície terrestre, expressas pelo relevo, são capazes de provocar variações nos atributos do solo em magnitudes diferenciadas, dependentes, principalmente, de um local específico da paisagem. Sendo assim, o objetivo deste trabalho foi realizar a caracterização mineralógica de Latossolos em diferentes feições do relevo na região de Jaboticabal, SP. Foram abertas três trincheiras nas pedoformas convexa, linear e côncava e, em seguida, foram realizadas a descrição morfológica dos horizontes e coleta de solo para caracterização física, química e mineralógica. Constatou-se que a mineralogia oxídica é dependente do tipo de pedoforma, com dominância de goethita na pedoforma côncava e a hematita dominou em pedoforma linear. Os minerais da fração argila desferrificada exibiram domínio de gibbsita na pedoforma côncava, em comparação as pedoformas convexas e lineares que predominam a mineralogia caulinítica.
\end{abstract}

Palavras-chave - Forma da paisagem. Pedogênese. Mineralogia do solo.

\begin{abstract}
Different surface land form expressed by the relief can cause variations in soil properties in different magnitudes, depending mainly on a specific location in the landscape. Therefore, the objective of this study was to make the mineralogical characterization of Oxisols in different relief forms in the region of Jaboticabal, SP. Three trenches were opened in the convex, linear and concave landforms, done morphological description of the soil horizons and collected soil samples for physical, chemical and mineralogical characterization. It was found that the oxidic mineralogy is dependent on the type of landform, with the dominance of goethite on the concave landform and hematite on the linear landform. The desferrific clay minerals exhibited dominance of gibbsite in the concave, compared to convex and linear landforms that were dominated by the kaolinitic mineralogy.
\end{abstract}

Key words - Landscape form. Pedogenesis. Soil mineralogy.

\footnotetext{
* Autor para correspondência

${ }^{1}$ Recebido para publicação em 12/07/2009; aprovado em 30/04/2010

Parte da Dissertação do primeiro autor apresentada no Programa de Pós-Graduação em Ciência do Solo da FCAV/UNESP

${ }^{2}$ Universidade Federal do Mato Grosso do Sul, Campus de Chapadão do Sul, Chapadão do Sul-MS, Brasil, rafamontana@hotmail.com

${ }^{3}$ Departamento de Solos e Adubos, FCAV/UNESP, Jaboticabal-SP,Brasil, marques@fcav.unesp.br

${ }^{4}$ Colegiado de Agronomia, IEAA/UFAM, Humaitá-AM, Brasil, mcesarsolos@gmail.com

${ }^{5}$ Departamento de Água e Solo, FEAGRI/UNICAMP, Campinas-SP, Brasil, zigomams@agr.unicamp.br

${ }^{6}$ Programa de Pós-Graduação em Produção Vegetal, FCAV/UNESP, Jaboticabal-SP, Brasil, li_arantes@yahoo.com.br
} 


\section{Introdução}

As diferentes feições da superfície terrestre, expressas pelo relevo em várias escalas, são capazes de provocar variações nos atributos do solo em magnitudes diferenciadas, dependentes principalmente de um local especifico da paisagem (SCHOORL et al., 2000). Em relação aos atributos mineralógicos, Curi e Franzmeier (1984) afirmam que o teor e os atributos cristalográficos dos minerais da fração argila são fortemente influenciados pelas características pedoambientais. Nesse sentido, Kämpf e Curi (2000), estudando o comportamento dos óxidos e hidróxidos de ferro e alumínio, afirmam que estes são importantes indicadores pedogenéticos por terem sua formação influenciada pelas condições do ambiente e por persistirem por longo tempo no solo.

As características dos ambientes em que são formados os minerais da fração argila explicam em grande parte a ocorrência de populações mineralógicas distintas ou não. Neste caso, as feições do relevo, o material de origem e o regime hídrico, como importantes componentes ambientais, imprimem variação ao sistema(CAMPOS etal., 2007a). De acordo Ghidin et al. (2006), no caso do relevo, suas influências são determinantes, pois este interfere no comportamento da água no solo e conseqüentemente promoção de reações químicas e transporte de sólidos ou de materiais em solução, produzindo efeitos diretos nos atributos mineralógicos.

Sabendo-se que a mineralogia dos solos é resultante da integração dos processos e fatores de formação, muitos autores têm estudado o relevo como fator condicionador de teores e cristalinidade dos minerais da fração argila. Camargo et al. (2008), estudando os atributos mineralógicos da fração argila de um Latossolo sob diferentes formas do relevo na região de Guaíba, SP, verificou maiores diâmetro médio do cristal para goethita, hematita e gibbsita nas pedoformas convexo-côncavas, indicando maior grau de cristalinidade quando comparado as pedoformas lineares. Já Curi e Franzmeier (1984), estudando uma toposseqüência de Latossolos desenvolvidos de basaltos no Planalto Central (Goiás), encontraram a maior concentração de gibbsita na posição mais elevada de uma toposseqüência e maior concentração de caulinita nas posições mais baixas, associadas à goethitas de menor diâmetro médio do cristal.

Entretanto, conforme destaca Berg e Oliveira (2000), no caso do Brasil, que apresenta vasto território com presença de Latossolos, os quais são muitas vezes sub-explorados surge a necessidade de um maior aprofundamento no estudo da distribuição de seus atributos mineralógicos associados às pequenas variações do relevo.
Dessa forma, buscando elucidar estes questionamentos, o objetivo deste trabalho foi caracterizar a mineralogia de Latossolos em diferentes feições do relevo, na região de Jaboticabal, SP.

\section{Material e métodos}

\section{Área de estudo}

As áreas de estudo localizam-se no município de Jaboticabal (SP), nordeste do Estado de São Paulo, as quais estão inseridas no Planalto Ocidental Paulista $\left(21^{\circ} 17^{\prime}\right.$ a $21^{\circ} 18^{\prime}$ de latitude sul e $48^{\circ} 08^{\prime}$ a $48^{\circ} 10^{\prime}$ de longitude oeste). O relevo é predominantemente suave ondulado a ondulado com altitude média de $600 \mathrm{~m}$. Nessa área, predominam os Latossolos distribuídos ao longo das vertentes, com perfis retilíneos e convexos cujo material de origem consiste principalmente de arenitos da Formação Adamantina.

O clima é classificado, segundo Köppen, como subtropical com inverno seco (Cwa), com precipitação média anual de aproximadamente $1.600 \mathrm{~mm}$, com distribuição concentrada no período de outubro a março e relativa seca no período de abril a setembro.

Realizou-se o mapeamento semi-detalhado de uma área de 158 hectares, utilizando equipamento de sistema de posicionamento global de navegação (GPS). Em seguida, com as coordenadas geográficas (altitude, latitude e longitude), elaborou-se o Modelo Digital de Elevação (MDE) da área de estudo cuja fisiografia é representativa da região (Figura1).

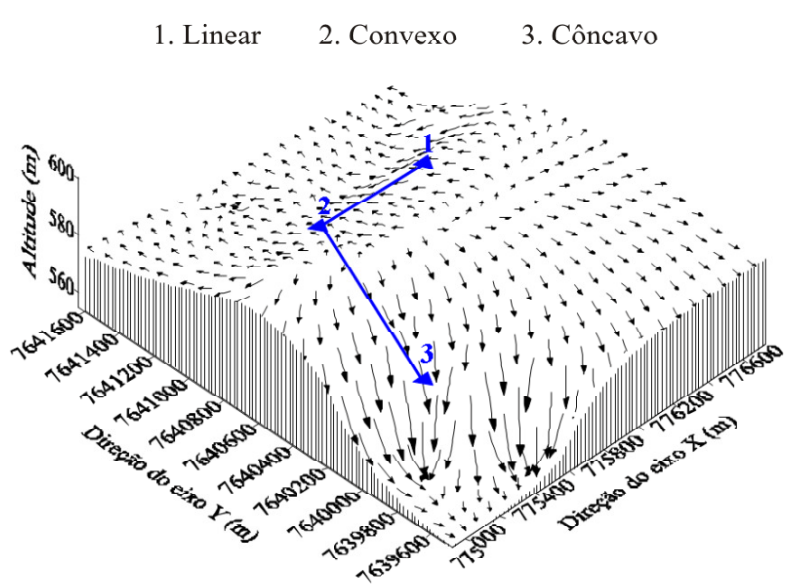

Figura 1 - Modelo de elevação digital das áreas estudadas, localização das trincheiras $(\nabla)$ e direcionamento do fluxo de água $(\rightarrow)$ 
Foram abertas três trincheiras seguindo o transecto ilustrado Figura 1, com presença de Latossolos nas pedoformas convexa, linear e côncava conforme o modelo de Troeh (1965). A identificação dos horizontes, a descrição morfológica e a coleta de amostras dos horizontes dos solos foram realizadas conforme Santos et al. (2005). Os solos foram classificados segundo critérios estabelecidos pelo SiBCS (EMBRAPA, 2006).

\section{Análises físicas}

A granulometria foi realizada pelo método da pipeta, empregando uma solução de $\mathrm{NaOH} 0,1 \mathrm{~N}$ como dispersante químico e agitação mecânica em aparato de baixa rotação por $16 \mathrm{~h}$, seguindo método modificado da (EMBRAPA, 1997). A fração argila foi separada por sedimentação gravitacional; a areia grossa e fina, por tamisação; e o silte, calculado por diferença.

\section{Análises químicas}

Cálcio, magnésio, e potássio trocáveis e fósforo $\mathrm{P}$ disponível foram extraídos utilizando-se o método da resina trocadora de íons (RAIJ et al., 2001); a matéria orgânica foi determinada segundo (EMBRAPA, 1997). Com base nos resultados das análises químicas, foram calculadas as somas de bases (SB), a capacidade de troca catiônica (CTC) e a saturação por bases (V).

$\mathrm{O} \mathrm{pH}$ foi determinado potenciometricamente, utilizando-se a relação 1:2,5 de solo: água e $\mathrm{KCl}$ (EMBRAPA, 1997).

Os óxidos do ataque sulfúrico $\left(\mathrm{SiO}_{2}, \mathrm{Al}_{2} \mathrm{O}_{3}, \mathrm{Fe}_{2} \mathrm{O}_{3}\right)$ foram determinados segundo o método proposto pela (EMBRAPA, 1997). Os óxidos de ferro livres foram extraídos com ditionito-citrato-bicarbonato (MEHRA; JACKSON, 1960); o ferro mal cristalizado foi extraído com oxalato ácido de amônio, segundo Camargo et al. (1986).

\section{Atributos mineralógicos \\ Óxidos de ferro}

A concentração dos óxidos de ferro foi realizada em amostras pulverizadas após tratamento da fração argila com NaOH $5 \mathrm{~mol} \mathrm{~L}^{-1}$, segundo método de Norrish e Taylor (1961), modificado por Kämpf e Schwertmann (1982). Para a manutenção de concentração mínima de acido silícico, na solução de $\mathrm{NaOH} 5 \mathrm{~mol} \mathrm{~L}^{-1}$, foram adicionados $10 \% \mathrm{em}$ peso de sílica gel moída (KÄMPF; SCHWERTMANN, 1982). Em seguida, as amostras foram lavadas com solução de $\mathrm{HCl}$ 0,5 mol L ${ }^{-1}$ em agitação por 4 horas. Para a correção dos desvios no posicionamento (d) dos reflexos, foi acrescentado às amostras $10 \%$ em peso de $\mathrm{NaCl}$ moído e peneirado.

A difração dos raios-X foi realizada em aparelho HGZ, equipado com cátodo de cobalto, filtro de ferro, radiação $\mathrm{K}(20 \mathrm{~mA}, 30 \mathrm{kV})$ e a velocidade de varredura igual a $1^{\circ} 2 \theta \mathrm{min}^{-1}$, empregados na difração de raios $\mathrm{X}$. Todas as amostras foram preparadas pelo método do pó e peneiradas em malha $0,10 \mathrm{~mm}$. Utilizou-se $\mathrm{NaCl}$ como padrão interno na correção das distorções instrumentais (posição e largura à meia altura dos picos).

A razão $\mathrm{Gt} /(\mathrm{Gt}+\mathrm{Hm})$ foi obtida após o cálculo das áreas dos reflexos da Hm (012) e Gt (110) nos reflexos dos difratogramas e, nesse caso, foi multiplicada a área da Gt (110) pelo valor 0,35, devido a intensidade de 35\% da Hm (012) (KÄMPF; SCHWERTMANN, 1998). Para quantificar a $\mathrm{Hm}$ e Gt na fração argila, promoveuse a alocação do $\mathrm{Fe}_{2} \mathrm{O}_{3}$ obtido com DCB nestes minerais, considerando a fórmula química, relação $\mathrm{Gt} /(\mathrm{Gt}+\mathrm{Hm})$ e nível de substituição isomórfica de $\mathrm{Fe}$ por $\mathrm{Al}$ na estrutura. A substituição isomórfica (SI) de Fe por Al na estrutura da $\mathrm{Hm}$ e Gt foi estimada pela posição dos picos destes minerais. A posição dos picos de $\mathrm{NaCl}$, em cada amostra, foi utilizada para corrigir os valores das posições dos picos da Hm e Gt. A SI na Gt foi calculada, segundo Schulze (1984), e, na Hm, segundo Schwertmann et al. (1979).

O Diâmetro Médio do Cristal (DMC) foi calculado com base na largura à meia altura (LMA) e na posição dos reflexos dos minerais Hm (110) e Gt (110). A LMA foi corrigida com o uso de amostras de itabirito, hematita e quartzo moídas e peneiradas $(0,1 \mathrm{~mm})$ (SCHULZE, 1984), utilizando-se a equação de Scherrer (KLUG; ALEXANDER, 1954).

\section{Caulinita - gibbsita}

O sistema caulinita-gibbsita foi obtido pelo método de análise da fração argila desferrificada, por difração de raios X, pelo método do pó (CAMARGO et al., 1986). $\mathrm{O}$ difratômetro utilizado foi o HZG-4/B, empregando-se cátodo de $\mathrm{Cu}$ com filtro de $\mathrm{Ni}$ e radiação $\mathrm{K}(20 \mathrm{~mA}, 30 \mathrm{Kv})$, com velocidade de varredura empregada de $1^{\circ} 2 \theta \mathrm{min}^{-1}$. A relação $\mathrm{Ct} /(\mathrm{Ct}+\mathrm{Gb})$ foi calculada empregando-se as áreas dos reflexos $\mathrm{Ct}$ (001) e Gb (002).

O DMC da Gb e da Ct foi calculado a partir da LMA das reflexões 002 e 001, respectivamente, em amostra desferrificada (tratadas com DCB). De maneira análoga aos óxidos de $\mathrm{Fe}$, o DMC foi obtido pela equação de Scherrer.

\section{Resultados e discussão}

\section{Atributos morfológicos}

Exceto os horizontes $\mathrm{A}_{1}$ e $\mathrm{AB}$ da pedoforma convexa que apresentaram cores com matiz $5 \mathrm{YR}$, todos os demais horizontes dos perfis dos solos apresentaram cores vermelhas, com matiz de 2,5YR (Tabela 1), 
indicativo de presença predominante de mineralogia oxídica, resultados semelhantes foram encontrados por Centurion et al. (1995), estudando Latossolos em Jaboticabal.

A classe textural no horizonte superficial de todos os perfis é franco-argilo-arenosa e, no horizonte B, latossólico franco-argilo-arenosa, passando por argiloarenosa e chegando a argila (Tabela 1). O domínio da fração areia nos perfis é explicado pelo material de origem, constituído de arenitos da Formação Adamantina. Destaca-se, entretanto, o aumento da fração argila em profundidade na pedoforma linear, localizada em posição de melhor drenagem na encosta, demonstrando incipiente processo de argiluvização.

Quanto à estrutura, observa-se que todos os horizontes dos solos estudados apresentaram estrutura granular variando de grande a muito pequena (Tabela 1) o que, segundo Resende et al. (1995), é característica de horizontes B latossólicos.

\section{Atributos físicos}

Quanto à composição granulométrica (Tabela 2), houve uma tendência de aumento dos teores de argila em profundidade, principalmente, nas pedoformas linear e convexa ( $\mathrm{P} 1$ e $\mathrm{P} 2)$, enquanto que na pedoforma côncava (P3) houve uma certa variação nos teores de argila entre os horizontes, fato que possivelmente esteja associado a própria conformação do relevo que direciona o fluxo de água, favorecendo o carreamento e redistribuição de partículas finas no interior do solo, conforme destaca Souza et al. (2004), que estudou a influência das pedoformas no comportamento dos atributos físicos de um Latossolo sob cultivo de canade-açúcar. Quanto aos teores da fração areia, verificouse que a fração areia grossa é pequena em todos os horizontes, fato explicado pelo material origem, pois os arenitos do grupo Bauru, Formação Adamantina apresentam presença majoritária da fração areia fina, corroborando com os resultados encontrado por Cunha et al. (2005) em estudo de uma toposseqüência na região de Jaboticabal, SP.

A relação silte/argila é um índice que auxilia na mensuração do grau de intemperização dos solos, conforme destaca Anjos et al., (1998). No estudo em questão, verificou-se uma tendência de homogeneidade em todas as pedoformas estudadas, com valores variando entre 0,10 e 0,24 (Tabela 2).

Tabela 1 - Principais atributos morfológicos dos Latossolos nas diferentes pedoformas

\begin{tabular}{|c|c|c|c|c|}
\hline \multirow{2}{*}{ Horizonte } & Profundidade & Cor Munsell & Textura & \multirow{2}{*}{ Estrutura $^{2}$} \\
\hline & $\mathrm{cm}$ & (úmida) & & \\
\hline \multicolumn{5}{|c|}{ Pedoforma Linear - P1 - Latossolo Vermelho distrófico típico } \\
\hline $\mathrm{A}_{1}$ & $0-16$ & $2,5 Y R 3 / 4$ & Franco-argilo-arenosa & fo., med. a gr., gran \\
\hline $\mathrm{AB}$ & $16-60$ & $2,5 Y R 4 / 4$ & Argilo-arenosa & fo., med. a gr., gran \\
\hline $\mathrm{B}_{\mathrm{W} 1}$ & $60-97$ & $2,5 \mathrm{YR} 4 / 3$ & Argila & fo., gr., gran \\
\hline $\mathrm{B}_{\mathrm{w} 2}$ & $97-151$ & $2,5 \mathrm{YR} 4 / 4$ & Argila & fo., med. a gr., gran \\
\hline $\mathrm{B}_{\mathrm{w} 3}$ & $151+$ & 2,5YR 3/4 & Argila & fo., med. a gr., gran \\
\hline \multicolumn{5}{|c|}{ Pedoforma Convexa - P2 - Latossolo Vermelho distrófico típico } \\
\hline $\mathrm{A}_{1}$ & $0-27$ & $5 Y R 4 / 4$ & Franco-argilo-arenosa & fo., méd a gr., gran. \\
\hline $\mathrm{AB}$ & $27-61$ & $5 Y R 4 / 4$ & Franco-argilo-arenosa & fo., peq., gran. \\
\hline $\mathrm{BA}$ & $61-93$ & $2,5 Y R 3 / 4$ & Franco-argilo-arenosa & fo., peq., gran. \\
\hline $\mathrm{B}_{\mathrm{w} 1}$ & $93-126$ & $2,5 \mathrm{YR} 4 / 4$ & Franco-argilo-arenosa & mod., gr. a mgr., gran. \\
\hline $\mathrm{B}_{\mathrm{w} 2}$ & $126-162$ & $2,5 \mathrm{YR} 4 / 4$ & Franco-argilo-arenosa & mod., méd. a peq., gran. \\
\hline \multicolumn{5}{|c|}{ Pedoforma Côncava - P3 - Latossolo Vermelho distrófico cambissólico } \\
\hline $\mathrm{A}_{1}$ & $0-28$ & $2,5 \mathrm{YR} 3 / 4$ & Franco-argilo-arenosa & fo., peq., gran. \\
\hline $\mathrm{AB}$ & $28-63$ & $2,5 \mathrm{YR} 3 / 4$ & Argilo-arenosa & fo., med. a gr., gran \\
\hline $\mathrm{BA}$ & $63-98$ & $2,5 Y R 3 / 4$ & Argilo-arenosa & fo., peq., gran. \\
\hline $\mathrm{B}_{\mathrm{W} 1}$ & $98-133$ & $2,5 \mathrm{YR} 4 / 4$ & Argilo-arenosa & fo., peq., gran. \\
\hline $\mathrm{B}_{\mathrm{W} 2}$ & $133+$ & $2,5 \mathrm{YR} 4 / 4$ & Argilo-arenosa & mod., peq., gran. \\
\hline
\end{tabular}

${ }^{2}$ fr.: fraca, mod.: moderada; fo.:forte; gran.: granular; peq.: pequena, méd.: média: gr.: grande; mgr.: muito grande 
Tabela 2 - Composição granulométrica dos Latossolos nas diferentes pedoformas

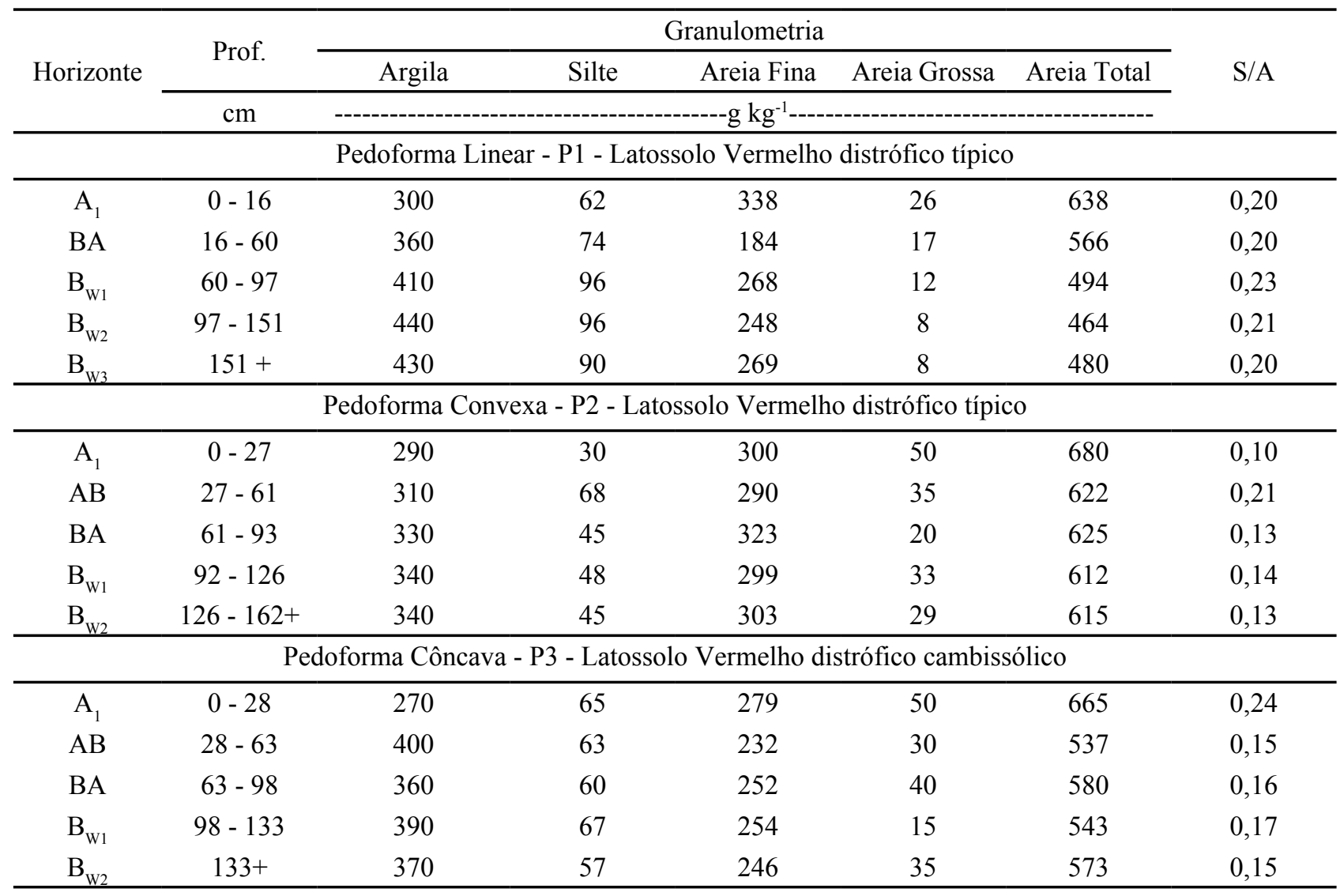

Os perfis apresentam classe de reação do solo ácida, com valores de $\mathrm{pH}$ em água variando entre 4,0 e 6,2 (Tabela 3). Observa-se que nos horizontes $\mathrm{Bw}_{1} \mathrm{e}$ $\mathrm{Bw}_{2}$ de todas as pedoformas estudadas há uma tendência de aumento nos valores de $\mathrm{pH}$ tanto em água como em $\mathrm{KCl}$, porém com valores mais elevados (variando entre 5,9 e 6,2) no perfil da pedoforma côncava. Este fato deve-se, possivelmente, à conformação do relevo, que promove a movimentação convergente da água favorecendo a migração, dissolução e percolação de constituintes minerais e orgânicos, conforme destaca Campos et al. (2007b).

Os teores de matéria orgânica não variaram em função das formas do relevo e, de maneira geral, observase valores mais pronunciados no horizonte superficial, fato associado à cobertura vegetal. Quanto à soma de bases (SB), observa-se que os perfis das pedoformas linear e convexa apresentam comportamento semelhante com diminuição em profundidade, enquanto que o perfil da pedoforma côncava apresentou padrão de comportamento contrário (Tabela 3), indicativo da interferência do fluxo da água no comportamento do atributo do solo que, segundo Rawls e Pachepsky (2002), as formas côncavas favorecem maior remoção de componente (minerais e orgânicos) das partes mais inclinadas e deposição nas posições mais estáveis da paisagem.

Quanto aos valores V\%, estes apresentaram comportamento similar aos da soma de bases. Todos os perfis apresentaram baixa saturação por bases $(\mathrm{V}<50 \%)$ nos horizontes diagnósticos subsuperficiais, identificando o seu caráter distrófico (EMBRAPA, 2006). Já a acidez potencial $(\mathrm{H}+\mathrm{Al})$ e capacidade de troca catiônica (CTC) diminuem com a profundidade (Tabela 3), corroborando com os resultados encontrados por Coelho e Vidal-Torrado (2003), que afirmam que solos desenvolvidos a partir de arenito da Formação Adamantina apresentam menor acidez potencial e CTC potencial em profundidade.

A composição química da fração terra fina, determinada a partir do ataque sulfúrico, é apresentada na Tabela 4. Os resultados de $\mathrm{Ki}$ observados em todos os horizontes dos perfis estudados são inferiores a 2,0, corroborando com os resultados encontrados por Campos et al. (2007a), que estudaram solos desenvolvidos de arenitos na região de Pereira Barreto, SP. 
Tabela 3 - Atributos químicos e teor de matéria orgânica dos Latossolos nas diferentes pedoformas

\begin{tabular}{|c|c|c|c|c|c|c|c|c|c|c|c|}
\hline \multirow{2}{*}{ Horizonte } & \multirow{2}{*}{$\mathrm{pH} \mathrm{H}_{2} \mathrm{O}$} & \multirow{2}{*}{$\mathrm{pH} \mathrm{KCl}$} & \multirow{2}{*}{$\Delta \mathrm{pH}$} & $\mathrm{MO}$ & $\mathrm{Ca}$ & $\mathrm{Mg}$ & $\mathrm{K}$ & $\mathrm{H}+\mathrm{Al}$ & $\mathrm{SB}$ & CTC & $\mathrm{V}$ \\
\hline & & & & $\mathrm{g} \mathrm{kg}^{-1}$ & \multicolumn{6}{|c|}{ 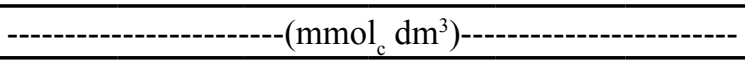 } & $\%$ \\
\hline \multicolumn{12}{|c|}{ Pedoforma Linear - P1 - Latossolo Vermelho distrófico típico } \\
\hline $\mathrm{A}_{1}$ & 5,9 & 4,6 & $-1,27$ & 11,0 & 13,0 & 8,0 & 4,5 & 31,0 & 25,5 & 56,5 & 45 \\
\hline $\mathrm{BA}$ & 4,7 & 4,0 & $-0,71$ & 10,0 & 5,0 & 3,0 & 2,5 & 42,0 & 10,5 & 52,5 & 20 \\
\hline $\mathrm{B}_{\mathrm{W} 1}$ & 4,3 & 4,1 & $-0,20$ & 7,0 & 5,0 & 2,0 & 1,1 & 42,0 & 8,1 & 50,1 & 16 \\
\hline $\mathrm{B}_{\mathrm{w} 2}$ & 4,6 & 4,2 & $-0,45$ & 5,0 & 4,0 & 2,0 & 0,5 & 34,0 & 6,5 & 40,5 & 16 \\
\hline $\mathrm{B}_{\mathrm{w} 3}$ & 5,3 & 4,2 & $-1,15$ & 5,0 & 5,0 & 1,0 & 0,5 & 34,0 & 6,5 & 40,5 & 16 \\
\hline \multicolumn{12}{|c|}{ Pedorforma Convexa - P2 - Latossolo Vermelho distrófico típico } \\
\hline $\mathrm{A}_{1}$ & 5,0 & 4,1 & $-0,92$ & 12,0 & 4,0 & 3,0 & 1,5 & 42,0 & 8,5 & 50,5 & 17 \\
\hline $\mathrm{AB}$ & 4,6 & 4,0 & $-0,58$ & 7,0 & 2,0 & 1,0 & 0,9 & 34,0 & 3,9 & 37,9 & 10 \\
\hline BA & 4,5 & 4,1 & $-0,35$ & 7,0 & 4,0 & 2,0 & 0,6 & 31,0 & 6,6 & 37,6 & 18 \\
\hline $\mathrm{B}_{\mathrm{W} 1}$ & 4,6 & 4,2 & $-0,43$ & 6,0 & 1,0 & 1,0 & 0,6 & 28,0 & 2,6 & 30,6 & 8 \\
\hline $\mathrm{B}_{\mathrm{w} 2}$ & 4,7 & 4,3 & $-0,34$ & 5,0 & 1,0 & 1,0 & 0,5 & 28,0 & 2,5 & 30,5 & 8 \\
\hline \multicolumn{12}{|c|}{ Pedoforma Côncava - P3 - Latossolo Vermelho distrófico cambissólico } \\
\hline$A_{1}$ & 5,0 & 4,2 & $-0,81$ & 16,0 & 6,0 & 4,0 & 1,2 & 47,0 & 11,2 & 58,2 & 19 \\
\hline $\mathrm{AB}$ & 4,4 & 4,1 & $-0,31$ & 10,0 & 2,0 & 2,0 & 0,8 & 42,0 & 4,8 & 46,8 & 10 \\
\hline BA & 5,8 & 5,4 & $-0,45$ & 7,0 & 9,0 & 8,0 & 1,0 & 18,0 & 18,0 & 36,0 & 50 \\
\hline $\mathrm{B}_{\mathrm{W} 1}$ & 6,2 & 5,9 & $-0,28$ & 7,0 & 10,0 & 5,0 & 0,7 & 18,0 & 15,7 & 33,7 & 47 \\
\hline $\mathrm{B}_{\mathrm{w} 2}$ & 6,2 & 6,1 & $-0,09$ & 6,0 & 9,0 & 4,0 & 0,5 & 13,0 & 13,5 & 26,5 & 51 \\
\hline
\end{tabular}

Tabela 4 - Óxidos silício, alumínio e ferro total $\left(\mathrm{Fe}_{\mathrm{t}}\right)$, índices Ki e Kr, ferro cristalino $\left(\mathrm{Fe}_{\mathrm{d}}\right)$ e amorfo $\left(\mathrm{Fe}_{\mathrm{o}}\right)$ e as relações $\mathrm{Fe}_{\mathrm{o}} / \mathrm{Fe}_{\mathrm{d}}$ e $\mathrm{Fe}_{\mathrm{d}} / \mathrm{Fe}_{\mathrm{t}}$ dos perfis estudados nas diferentes pedoformas

\begin{tabular}{|c|c|c|c|c|c|c|c|c|c|}
\hline \multirow{2}{*}{ Horizonte } & \multirow[t]{2}{*}{$\mathrm{SiO}_{2}$} & \multirow{2}{*}{$\frac{\mathrm{Al}_{2} \mathrm{O}_{3}}{\mathrm{~g} \mathrm{~kg}^{-1}}$} & $\mathrm{Fe}_{2} \mathrm{O}_{3}$ & \multirow{2}{*}{$\mathrm{Ki}$} & \multirow{2}{*}{$\mathrm{Kr}$} & $\mathrm{Fe}_{\mathrm{d}}$ & $\mathrm{Fe}_{\mathrm{o}}$ & \multirow{2}{*}{$\mathrm{Fe}_{\mathrm{o}} / \mathrm{Fe}_{\mathrm{d}}$} & \multirow{2}{*}{$\mathrm{Fe}_{\mathrm{d}} / \mathrm{Fe}_{\mathrm{t}}$} \\
\hline & & & & & & \multicolumn{2}{|c|}{$\mathrm{g} \mathrm{kg}^{-1}$} & & \\
\hline \multicolumn{10}{|c|}{ Pedoforma Linear - P1 - Latossolo Vermelho distrófico típico } \\
\hline $\mathrm{A}_{1}$ & 71,50 & 90,00 & 51,00 & 1,30 & 1,00 & 43,10 & 7,00 & 0,16 & 0,85 \\
\hline $\mathrm{AB}$ & 103,00 & 100,00 & 56,00 & 1,03 & 1,30 & - & - & - & - \\
\hline $\mathrm{B}_{\mathrm{w} 1}$ & 124,00 & 125,00 & 77,00 & 1,80 & 1,25 & - & - & - & - \\
\hline $\mathrm{B}_{\mathrm{w} 2}$ & 130,00 & 140,00 & 74,00 & 1,60 & 1,23 & 57,50 & 7,50 & 0,13 & 0,78 \\
\hline $\mathrm{B}_{\mathrm{w}_{3}}$ & 133,00 & 140,00 & 71,00 & 1,60 & 1,29 & - & - & - & - \\
\hline \multicolumn{10}{|c|}{ Pedoforma Convexa - P2 - Latossolo Vermelho distrófico típico } \\
\hline $\mathrm{A}_{1}$ & 69,50 & 85,00 & 53,00 & 1,40 & 1,00 & 36,40 & 6,30 & 0,17 & 0,69 \\
\hline $\mathrm{AB}$ & 87,00 & 100,00 & 50,00 & 1,50 & 1,16 & - & - & - & - \\
\hline $\mathrm{BA}$ & 91,50 & 115,00 & 51,00 & 1,30 & 1,07 & - & - & - & - \\
\hline $\mathrm{B}_{\mathrm{w} 1}$ & 97,00 & 120,00 & 54,00 & 1,40 & 1,14 & - & - & - & - \\
\hline $\mathrm{B}_{\mathrm{w} 2}$ & 106,50 & 125,00 & 57,00 & 1,50 & 1,33 & 45,60 & 6,60 & 0,15 & 0,80 \\
\hline \multicolumn{10}{|c|}{ Pedoforma Côncava - P3 - Latossolo Vermelho distrófico cambissólico } \\
\hline $\mathrm{A}_{1}$ & 68,50 & 110,00 & 67,00 & 1,10 & 0,77 & 45,50 & 6,00 & 0,13 & 0,68 \\
\hline $\mathrm{AB}$ & 79,50 & 125,00 & 70,00 & 1,10 & 1,07 & - & - & - & - \\
\hline BA & 61,00 & 135,00 & 72,00 & 0,80 & 0,57 & - & - & - & - \\
\hline $\mathrm{B}_{\mathrm{w} 1}$ & 82,00 & 140,00 & 76,00 & 1,00 & 0,76 & - & - & - & - \\
\hline $\mathrm{B}_{\mathrm{w}_{2}}$ & 85,50 & 145,00 & 78,00 & 1,00 & 0,74 & 57,80 & 7,00 & 0,12 & 0,74 \\
\hline
\end{tabular}


$\mathrm{Na}$ Tabela 4, são apresentados os valores de $\mathrm{Fe}_{\mathrm{o}}$, $\mathrm{Fe}_{\mathrm{d}}$ e $\mathrm{Fe}_{\mathrm{t}}$ e das relações estabelecidas entre as diferentes formas de Fe extraídas. Em todos os perfis, os teores de $\mathrm{Fe}_{\mathrm{d}}$ e $\mathrm{Fe}_{\mathrm{t}}$ são maiores nos horizontes B latossólicos $\left(\mathrm{Bw}_{2}\right)$. Por outro lado, os teores de ferro livre $\left(\mathrm{Fe}_{\mathrm{d}}\right)$ predominam sobre as formas de ferro mal cristalizadas $\left(\mathrm{Fe}_{\mathrm{o}}\right)$ para todos os horizontes diagnósticos superficiais e subsuperficiais dos perfis estudados, independente das formas do relevo.

A relação $\mathrm{Fe}_{\mathrm{o}} / \mathrm{Fe}_{\mathrm{d}}$ apresentou os maiores valores nos horizontes superficiais para todos os perfis estudados (Tabela 4), evidenciando presença de maiores teores do ferro de baixa cristalinidade, fato que está ligado principalmente ao conteúdo de matéria orgânica que favorece a inibição da cristalização do ferro pedogênico (SILVA et al., 2001). Por outro lado, verifica-se que os maiores valores da razão $\mathrm{Fe}_{\mathrm{o}} / \mathrm{Fe}_{\mathrm{d}}$ são encontrados nas pedoformas linear e convexa, indicando um menor grau de cristalinidade dos óxidos de ferro nestes solos, quando comparado a pedoforma côncava.

A relação $\mathrm{Fe}_{\mathrm{d}} / \mathrm{Fe}_{\mathrm{t}}$ é um índice auxiliar usado para estimar o grau de desenvolvimento do solo. Se tomarmos os valores médios, verifica-se que os maiores resultados foram encontrados nos perfis da pedoforma linear e convexa, quando comparado a côncava (Tabela 4), mostrando que os dois primeiros solos ficaram maior tempo exposto à ação do intemperismo, portanto são pedogeneticamente mais evoluídos (MARQUES JÚNIOR; LEPSCH, 2000).

Os valores de largura à meia altura (LMA) para a hematita variaram entre 0,30 a $0,36{ }^{\circ} 2 \theta$ (Tabela 5), com maiores resultados nos horizontes $\mathrm{B}$ latossólicos das pedoformas linear e convexa. Para a goethita, os valores situaram-se entre 0,18 e $0,75^{\circ} 2 \theta$. Verifica-se, neste caso, que os valores na pedoforma côncava foram observado maiores em relação às demais pedoformas (Tabela 5). De maneira geral, o diâmetro médio do cristal (DMC) da hematita apresentou valores entre 52 e $91 \mathrm{~nm}$, enquanto a goethita exibiu valores mais baixos (17 nm a $50 \mathrm{~nm})$, independente da pedoforma onde os solos ocorreram. Os resultados de LMA e DMC indicam maior cristalinidade da hematita na pedoforma côncava, enquanto que as menores cristalinidades ocorreram nas pedoformas linear e convexa, respectivamente, corroborando com Pocay (2000).

A substituição isomórfica (SI) de ferro por alumínio na hematita variou de 0,07 a $11,39 \mathrm{~mol} \%$ e de 32,57 a $35,88 \mathrm{~mol} \%$ para a goethita. Estes resultados demonstram maior taxa de substituição isomórfica na goethita, independente da pedoforma (Tabela 5). Segundo Kämpf e Curi (2000), uma substituição isomórfica dessa natureza é atribuída a goethitas de ambientes que favorecem a baixa atividade de silício e alta de alumínio.

Em relação aos teores de hematita e goethita (Tabela 5), verifica-se que os maiores teores de goethita encontram-se na pedoforma côncava, indicando ser esse o ambiente preferencial de formação desse mineral, enquanto os maiores teores para hematita foram identificados nos solos da pedoforma linear. De acordo com Pocay (2000), estes resultados refletem as boas condições de drenagem propiciadas pela pedoforma linear quanto comparado ao ambiente de ocorrência da pedoforma côncava.

A relação $\mathrm{Gt} /(\mathrm{Gt}+\mathrm{Hm})$ se apresentou maior nas pedoformas convexas e côncavas (Tabela 5), estes resultados podem ser atribuídos aos maiores teores de goethita nos ambientes mais úmidos com restrições de

Tabela 5 - Atributos cristalográficos dos óxidos de ferro dos perfis estudados nas diferentes pedoformas

\begin{tabular}{|c|c|c|c|c|c|c|c|c|c|c|c|}
\hline \multirow{3}{*}{ Horizonte } & \multicolumn{2}{|c|}{ Área do pico } & \multicolumn{2}{|c|}{$\mathrm{LMA}^{1}$} & \multicolumn{2}{|c|}{$\mathrm{DMC}^{2}$} & \multicolumn{2}{|c|}{$\mathrm{SI}^{3}$} & \multicolumn{2}{|c|}{ Teor } & \multirow{3}{*}{$\begin{array}{c}\mathrm{Gt} / \\
(\mathrm{Gt}+\mathrm{Hm})\end{array}$} \\
\hline & $\begin{array}{l}{ }^{4} \mathrm{Hm} \\
(012)\end{array}$ & $\begin{array}{c}{ }^{5} \mathrm{Gt} \\
(110)\end{array}$ & $\begin{array}{l}\mathrm{Hm} \\
(110)\end{array}$ & $\begin{array}{c}\mathrm{Gt} \\
(110)\end{array}$ & $\begin{array}{l}\mathrm{Hm} \\
(110)\end{array}$ & $\begin{array}{c}\mathrm{Gt} \\
(110)\end{array}$ & $\begin{array}{c}\mathrm{Hm} \\
(110)\end{array}$ & $\begin{array}{c}\mathrm{Gt} \\
(110)\end{array}$ & $\mathrm{Hm}$ & Gt & \\
\hline & \multicolumn{2}{|c|}{$\mathrm{cm}^{2}$} & \multicolumn{2}{|c|}{${ }^{\circ} 2 \theta$} & \multicolumn{2}{|c|}{$\mathrm{nm}$} & \multicolumn{2}{|c|}{$\mathrm{mol} \%$} & \multicolumn{2}{|c|}{$\mathrm{g} \mathrm{kg}^{-1}$} & \\
\hline \multicolumn{12}{|c|}{ Pedoforma Linear - P1 - Latossolo Vermelho Distrófico típico } \\
\hline $\mathrm{A}_{1}$ & 0,47 & 0,19 & 0,31 & 0,18 & 79,92 & 29,28 & 7,21 & 34,36 & 53,92 & 8,57 & 0,12 \\
\hline $\mathrm{B}_{\mathrm{W} 2}$ & 0,62 & 0,16 & 0,34 & 0,18 & 64,43 & 27,41 & 1,42 & 36,26 & 80,68 & 8,08 & 0,08 \\
\hline \multicolumn{12}{|c|}{ Pedoforma Convexa - P2 - Latossolo Vermelho Distrófico típico } \\
\hline $\mathrm{A}_{1}$ & 0,59 & 0,18 & 0,33 & 0,45 & 52,58 & 50,32 & 11,39 & 35,88 & 32,91 & 5,55 & 0,09 \\
\hline$B_{w 2}$ & 0,66 & 1,40 & 0,34 & 0,43 & 64,42 & 39,04 & 0,07 & 32,41 & 37,42 & 30,89 & 0,42 \\
\hline \multicolumn{12}{|c|}{ Pedoforma Côncava - P3 - Latossolo Vermelho Distrófico cambissólico } \\
\hline $\mathrm{A}_{1}$ & 0,55 & 0,60 & 0,36 & 0,45 & 58,77 & 36,77 & 11,39 & 34,45 & 47,09 & 19,99 & 0,27 \\
\hline $\mathrm{B}_{\mathrm{W} 2}$ & 0,40 & 1,10 & 0,30 & 0,75 & 90,80 & 17,07 & 7,21 & 34,65 & 42,12 & 45,07 & 0,49 \\
\hline
\end{tabular}

${ }^{1} \mathrm{LMA}=$ Largura Meia Altura, ${ }^{2} \mathrm{DMC}=$ Diâmetro Médio do Cristal, ${ }^{3} \mathrm{SI}=$ Substituição Isomórfica, ${ }^{4} \mathrm{Hm}=$ Hematita, ${ }^{5} \mathrm{Gt}=$ Goethita 
drenagem (côncavo), corroborando com Fontes e Weed (1991), que estudaram o comportamento dos óxidos de ferro em Latossolos sob diversos materiais de origem.

A mineralogia da fração argila desferrificada é apresentada na Tabela 6 . Independente das pedoformas, a LMA da caulinita apresentou valores semelhantes (entre 0,45 e $0,60^{\circ} 2 \theta$ ), fato também observado para a gibbsita $\left(0,18\right.$ e $\left.0,30^{\circ} 2 \theta\right)$. Quanto ao DMC da caulinita, os valores variaram entre 19,7 e 31,1 nm, enquanto para a gibbsita os valores variaram entre 74,03 e 81,43 nm. Estes resultados corroboram com Camargo et al. (2008), que estudou a mineralogia da fração argila de solos da região de Guariba, SP e verificou que os componentes da fração argila desferrificada, em ambiente altamente intemperizado, não apresentam dependência com as formas do relevo.

A relação $\mathrm{Ct} /(\mathrm{Ct}+\mathrm{Gb})$ indica o predomínio da mineralogia gibsítica no solo da pedoforma côncava, enquanto nas pedoformas convexas e lineares dominam a mineralogia caulinítica, concordando com os valores de $\mathrm{Ki}$ (Tabela 3), pois solos com maior grau de desenvolvimento apresentam enriquecimento relativo de $\mathrm{Gb}$ em relação aos menos evoluídos. Estes resultados estão de acordo com os encontrados por Pedrotti et al. (2003).

Tabela 6 - Atributos cristalográficos do sistema caulinita-gibbsita dos perfis estudados nas diferentes pedoformas

\begin{tabular}{|c|c|c|c|c|c|c|c|}
\hline \multirow{2}{*}{ Horizonte } & \multicolumn{2}{|c|}{ Area do pico $\left(\mathrm{cm}^{2}\right)$} & \multicolumn{2}{|c|}{${ }^{1} \mathrm{LMA}\left({ }^{\circ} 2 \theta\right)$} & \multicolumn{2}{|c|}{${ }^{2} \mathrm{DMC}(\mathrm{nm})$} & \multirow{2}{*}{$\mathrm{Ct} /(\mathrm{Ct}+\mathrm{Gb})$} \\
\hline & ${ }^{3} \mathrm{Ct}-001$ & ${ }^{4} \mathrm{~Gb}-002$ & Ct-001 & Gb-002 & Ct-001 & Gb-002 & \\
\hline \multicolumn{8}{|c|}{ Pedoforma Linear - P1 - Latossolo Vermelho Distrófico típico } \\
\hline $\mathrm{A}_{1}$ & 2,16 & 0,46 & 0,60 & 0,22 & 19,72 & 232,71 & 0,82 \\
\hline $\mathrm{B}_{\mathrm{w} 2}$ & 1,35 & 0,39 & 0,45 & 0,18 & 31,10 & 81,43 & 0,77 \\
\hline \multicolumn{8}{|c|}{ Pedoforma Convexa - P2 - Latossolo Vermelho Distrófico típico } \\
\hline $\mathrm{A}_{1}$ & 2,34 & 0,39 & 0,45 & 0,22 & 31,10 & 232,71 & 0,85 \\
\hline $\mathrm{B}_{\mathrm{w} 2}$ & 1,92 & 0,60 & 0,45 & 0,30 & 31,05 & 74,45 & 0,76 \\
\hline \multicolumn{8}{|c|}{ Pedoforma Côncava - P3 - Latossolo Vermelho Distrófico câmbico } \\
\hline $\mathrm{A}_{1}$ & 1,20 & 1,04 & 0,45 & 0,30 & 31,10 & 74,37 & 0,53 \\
\hline $\mathrm{B}_{\mathrm{w} 2}$ & 1,11 & 1,00 & 0,45 & 0,30 & 31,05 & 74,45 & 0,52 \\
\hline
\end{tabular}

${ }^{1} \mathrm{LMA}=$ Largura Meia Altura, ${ }^{2} \mathrm{DMC}=$ Diâmetro Médio do Cristalito, ${ }^{3} \mathrm{Ct}=$ Caulinita, ${ }^{4} \mathrm{~Gb}=$ Gibsita

\section{Conclusões}

1. A mineralogia oxídica é dependente do tipo de pedoforma, com dominância de goethita na pedoforma côncavo em relação às pedoformas linear e convexa, já a hematita apresenta maiores teores em pedoforma lineares em comparação as pedoformas convexa e côncava;

2. Os minerais da fração argila deserrificada exibiram domínio de gibbsita na pedoforma côncava, em comparação as pedoformas convexas e lineares, dominam a mineralogia caulinítica.

\section{Referências}

ANJOS, L. H. et al. Landscape and pedogenesis of na OxisolInceptisol-Ultisol sequence in Southeastern Brazil. Soil Science Society of America Journal, v. 62, p. 1651-1658, 1998.
BERG, M. Van Den; OLIVEIRA, J. B. Variability of apparently homogeneous soilscapes in São Paulo Stade, Brazil. II. Quality of soil maps. Revista Brasileira de Ciência do Solo, v. 24, p.393-407, 2000 .

CAMARGO, L. et al. Variabilidade espacial de atributos mineralógicos de um latossolo sob diferentes formas do relevo. I - Mineralogia da fração argila. Revista Brasileira de Ciência do Solo, v. 32, n. 06, p. 2269-2277, 2008.

CAMARGO, O. A. et al. Métodos de análise química, mineralógica e física de solos do IAC. Campinas: Instituto Agronômico de Campinas, 1986. 94 p. (Boletim Técnico, 106).

CAMPOS, M. C. C. et al. Relações solo-paisagem em uma litosseqüência arenito-basalto na região de Pereira Barreto, SP. Revista Brasileira de Ciência do Solo, v. 31, n. 03, p.519-529, 2007a.

CAMPOS, M. C. C. et al. Variabilidade espacial da textura de solos de diferentes materiais de origem em Pereira Barreto, SP. Revista Ciência Agronômica, v. 38, n. 02, p. 149-157, 2007b. 
CENTURION, J. F. et al. Características de latossolos roxos desenvolvidos de rochas alcalinas e básicas de Jaboticabal, SP. Scientia Agrícola. v. 52, n. 02, p. 226-232, 1995.

COELHO, M. R.; VIDAL-TORRADO, P. Caracterização e gênese de perfis plínticos desenvolvidos de arenito do Grupo Bauru: II - mineralogia. Revista Brasileira de Ciência do Solo, v. 27, n. 03, p. 495-507, 2003.

CUNHA, P. et al. Superfícies geomórficas e atributos de latossolos em uma seqüência arenítico-basáltica da região de Jaboticabal (SP). Revista Brasileira de Ciência do Solo. v. 29, n. 01, p. 81-90, 2005.

CURI, N.; FRANZMEIER, D. P. Toposequence of Oxisols from the central plateau of Brazil. Soil Science Society American Journal, v. 48, p. 341-346, 1984.

EMPRESA BRASILEIRA DE PESQUISA AGROPECUÁRIA (EMBRAPA). Centro Nacional de Pesquisa de Solos. Manual de métodos de análise de solo. Rio de Janeiro: Embrapa, 1997. 212 p.

EMPRESA BRASILEIRA DE PESQUISA AGROPECUÁRIA (EMBRAPA). Centro Nacional de Pesquisa de Solos. Sistema Brasileiro de Classificação de Solos. Rio de Janeiro: Embrapa, 2006. 306 p.

FONTES, M. P. F.; WEED, S. B. Iron oxides in selected Brazilian oxisol: I. Mineralogy. Science Society of America Journal, v. 55, p. 1143-1149, 1991.

GHIDIN, A. A. et al. Toposseqüências de Latossolos originados de rochas basálticas no Paraná: I - mineralogia da fração argila. Revista Brasileira de Ciência do Solo. v. 30, n. 02, p. 293-306. 2006.

KÄMPF, N.; CURI, N. Óxidos de ferro: indicadores de atributos de ambientes pedogênicos e geoquímicos. In: NOVAIS, R. F.; ALVAREZ V., V. H.; SCHAEFER, C. E. G. R. (Ed.) Tópicos em Ciência do Solo. Viçosa. Sociedade Brasileira de Ciência do Solo, 2000. p. 107-138. 1 v.

KÄMPF, N.; SCHWERTMANN, U. Avaliação da estimativa de substituição de Fe por Al em hematitas de solos. Revista Brasileira de Ciência do Solo, v. 22, n. 02, p. 209-213, 1998.

KÄMPF, N.; SCHWERTMANN, U. Relações entre óxidos de ferro e cor em solos cauliníticos do Rio Grande do Sul. Revista Brasileira de Ciência do Solo, v. 07, p. 27-31, 1982.

KLUG, H. P.; ALEXANDER, L. E. X-ray diffraction procedures for polycrystalline and amorphous materials. New York: John Wiley \& Sons, 1954. 716 p.

MARQUES JÚNIOR, J.; LEPSCH, I. F. Depósitos superficiais neocenozóicos, superfícies geomórficas e solos em Monte Alto, SP. Geociência, v. 19, n. 02, p. 265-281, 2000.
MENHRA, O. P.; JACKSON, M. L. Iron oxide from soils and clays by a dithionite-citrate system buffered with sodium bicarbonate. Clays Mineralogy, v. 07, p. 317-327, 1960.

NORRISH, K.; TAYLOR, R. M. The isomorphous replacement of iron by aluminium in soil goethites. Soil Science Society of America Journal, v. 12, p. 294-306, 1961.

PEDROTTI, A. et al. Relação entre atributos físicos, mineralogia da fração argila e formas de alumínio no solo. Revista Brasileira de Ciência do Solo, v. 27, n. 01, p. 1-9, 2003.

POCAY, V. G. Relações entre pedoforma e variabilidade espacial de atributos de latossolos sob cultivo intensivo de cana-de-açúcar. 2000. 177 f. Dissertação (Mestrado em Ciência do Solo) - Faculdade de Ciências Agrárias e Veterinárias, Universidade Estadual de São Paulo.

RAIJ, B. Van et al. (Ed.) Análise química para avaliação da fertilidade de solos tropicais. Campinas: Instituto Agronômico de Campinas, 2001. 285 p.

RAWLS, W. J.; PACHEPSKY, Y. A. Using. field topographic to estimate soil water. retention. Soil Science, v. 167, p. 423435, 2002.

RESENDE, M. et al. Pedologia: base para distinção de ambientes. Viçosa: NEPUT, 1995. 304 p.

SANTOS, R. D. et al. Manual de descrição e coleta de solos no campo. 5. ed. Viçosa, MG, Sociedade Brasileira de Ciência do Solo, 2005. 100 p.

SCHOORL, J. M. et al. Three dimensional landscape process modeling: The effect of DEM resolution. Earth Surface Proc Landforms, v. 25, p. 1025-1034, 2000.

SCHULZE, D. G. The influence of aluminum on iron oxides: VIII. Unit-cell dimensions of Al-substituted goethites and estimation of $\mathrm{Al}$ from them. Clays and Clay Minerals, v. 32, $\mathrm{n}$. 01, p. 36-44, 1984.

SCHWERTMANN, U. et al. The influence of aluminum on iron oxides. Part II. Preparation and properties of Al-substituted hematites. Clays and Clay Minerals, v. 27, n. 02, p. 105-112, 1979.

SILVA, M. B. et al. Estudo de topossequência da baixada litorânea fluminense: efeitos do material de origem e posição topográfica. Revista Brasileira de Ciência do Solo, v. 25, n. 04, p. 965-976, 2001.

SOUZA, Z. M. et al. Influência da pedoforma na variabilidade espacial de alguns atributos físicos e hídricos de um Latossolo sob cultivo de cana-de-açúcar. Irriga, v. 09, n. 01, p. 1-11, 2004.

TROEH, F. R. Landform equations fitted to contour maps. Soil Science Society American Journal, v. 263, n. 07, p. 616-27, 1965. 